\title{
Neuromechanical and Environment Aware Machine Learning Tool for Human Locomotion Intent Recognition
}

\author{
Simão Carvalho, Joana Figueiredo, and Cristina P. Santos
}

\begin{abstract}
Current research suggests the emergent need to recognize and predict locomotion modes (LMs) and LM transitions to allow a natural and smooth response of lower limb active assistive devices such as prostheses and orthosis for daily life locomotion assistance. This Master dissertation proposes an automatic and user-independent recognition and prediction tool based on machine learning methods. Further, it seeks to determine the gait measures that yielded the best performance in recognizing and predicting several human daily performed LMs and respective LM transitions. The machine learning framework was established using a Gaussian support vector machine (SVM) and discriminative features estimated from three wearable sensors, namely, inertial, force and laser sensors. In addition, a neuro-biomechanical model was used to compute joint angles and muscle activations that were fused with the sensor-based features. Results showed that combining biomechanical features from the Xsens with environment-aware features from the laser sensor resulted in the best recognition and prediction of $\mathrm{LM}(\mathrm{MCC}=\mathbf{0 . 9 9}$ and $\mathrm{MCC}=\mathbf{0 . 9 5})$ and $\mathrm{LM}$ transitions $(\mathrm{MCC}=\mathbf{0 . 9 6}$ and $\mathrm{MCC}=\mathbf{0 . 9 8})$. Moreover, the predicted LM transitions were determined with high prediction time since their detection happened one or more steps before the LM transition occurrence. The developed framework has potential to improve the assistance delivered by locomotion assistive devices to achieve a more natural and smooth motion assistance.
\end{abstract}

\section{INTRODUCTION}

Due to the increasing integration of robotics in the field of medicine over the last few years, a larger number of people with locomotion disabilities are given a chance to live a life with better quality. According to the World Health Organisation, every year 15 million people suffer a stroke (20000 in Portugal) and 80 people in a million worldwide suffer a spinal cord injury ( 58 per million in Portugal), leaving people with motor disabilities [1], [2].

Advancements towards the use of assistive devices (e.g., active orthosis, exoskeletons and prosthesis) in daily life

*This work has been supported in part by the Fundação para a Ciência e Tecnologia (FCT) with the Reference Scholarship under Grant SFRH/BD/108309/2015, and part by the FEDER Funds through the Programa Operacional Regional do Norte and national funds from FCT with the project SmartOs -Controlo Inteligente de um Sistema Ortótico Ativo e Autónomounder Grant NORTE-01-0145-FEDER-030386, and by the FEDER Funds through the COMPETE 2020-Programa Operacional Competitividade e Internacionalização (POCI) — with the Reference Project under Grant POCI-010145-FEDER-006941.

Simão Carvalho is with University of Minho, researcher at Center for MicroElectroMechanical; e-mail: simaopedrocarvalho17@gmail.com).

Joana Figueiredo is with University of Minho, Ph.D. student at Center for MicroElectroMechanical; e-mail: id6003@alunos.uminho.pt).

Cristina P. Santos professor at University of Minho, Center for MicroElectroMechanical; e-mail: cristina@dei.uminho.pt). situations, as well as the ongoing integration of compliant robotic devices in neurorehabilitation generates new challenges. Firstly, the timely adaptation of these devices to diverse locomotion modes (LMs) such as, level-ground walking, climbing stairs and ramps. Secondly, to tune the assistance to the patient's locomotion intention by monitoring the human-robotic interface through wearable sensors [3], [4].

To provide efficient and suitable gait assistance and to decrease fall risk, LM recognition is essential. LM recognition is challenging for gait assistance devices due to the limited sensors and the need for a time effective response [5]. Commercially available devices such as prosthesis and orthosis can switch LMs executing manual commands such as button presses, as used in the ReWalkTM system (Argo Medical Technologies Ltd. Israel) [6] or specific motions such as used in Power KneeTM (Össur, Iceland) [6]. The user's intent motion prediction is fundamental to achieve a natural, user-independent and automatic device response during all locomotion phases. However, the existing manual solutions require the user's training before using the device and a major cognitive effort from the user during locomotion.

Previous studies have proposed promising methods to recognize and predict LMs and LM transitions, through artificial intelligence methods, using several wearable sensors. One approach proposed by Chen et. al. [7] exploited signals from two-foot pressure insoles and three inertial measurement units (IMU) as biomechanical features to develop a locomotion intent prediction strategy based in a linear discriminate classifier (LDA). Six kinds of LMs were accurately recognized $(99.71 \% \pm 0.05)$, however no LM prediction was addressed [7]. The electromyography signal (EMG) is another widely used measure for user's intent recognition during locomotion since it represents muscle activations that precede the limb's motion. Some studies addressed the sensor fusion between EMG and mechanical signals from the user's assistive device such as moment, velocity, angle, gait phase and ground reaction force to be used in machine learning algorithms such as LDA and Support Vector Machines (SVMs) [8], [9] These studies report an error lower than $2.3 \% \pm 0.7 \%$ for detecting 8 different LM transition.

Despite being widely used, wearable sensors such as IMUs and EMG present some major drawback such as the presence of noise, which introduces drift errors in IMUs 'measures [10]-[12]. Moreover, EMG-based approaches present some practical limitations, namely the EMG electrode shift due to the subject movement or sweat and require an expert and a lengthy installation [13], [14]. The EMG signal is extremely dependent on the electrode's position and orientation. Placing 
electrodes in the wrong locations will reduce signal strength and quality. Neuro-biomechanical models (combining biomechanics and neurophysiology) have provided accurate kinematic, dynamic estimations, and muscular activation estimations patterns using wearable sensor information such as IMUs and EMGs and resemble a promising method to overcome the mentioned issues[15], [16]

Research efforts have been made to further improve the motion intent recognition and prediction, including environment-aware strategies to infer about the interaction between the user and the world, inspired by the human ability to see the surrounding environment. In [17], an infrared laser sensor was placed in the user's waist in order to acquire terrain change information during the subject's gait. Study [17] proposed a fusion between an LM recognition module and a terrain reconstruction module, using an LDA classifier. The terrain reconstruction module provided an accuracy higher than $98 \%$ and detections $500 \mathrm{~ms}$ in advance. The anticipatory nature of environment-aware strategies still needs to be further explored to provide robust and time-effective LM transition prediction systems for timely controlling assistive devices.

This dissertation tackles the mentioned challenges. It proposes an automatic and user-independent recognition and prediction tool for classifying daily encountered LMs and LM transitions using machine learning methods. Furthermore, different types of wearable sensors, namely IMU and force sensing resistor sensors (biomechanical gait measure), and an infrared laser sensor (environment-aware gait measure), were explored to find an effective and reliable recognition and prediction tool. Additionally, the Xsens biomechanical model and an OpenSim modified musculoskeletal model are used to generate neuro-biomechanical features such as the lower limbs joint angles and muscle activation patterns, respectively. The tool aims at an efficient classification of the LMs commonly encountered in daily life, with variations in walking speed and terrains (i.e., flat, ascending and descending stairs, climbing up and down ramp). The tool also approaches transitions from/to those terrains using the user's self-selected lower limb to minimize the user's cognitive effort during locomotion. The validation of the proposed tool included 10 able body subjects performing different LMs and the LM transitions at different terrains, directions, and gait speeds. The obtained results may contribute to the optimization of the assistance delivered by the lower limb active assistive devices.

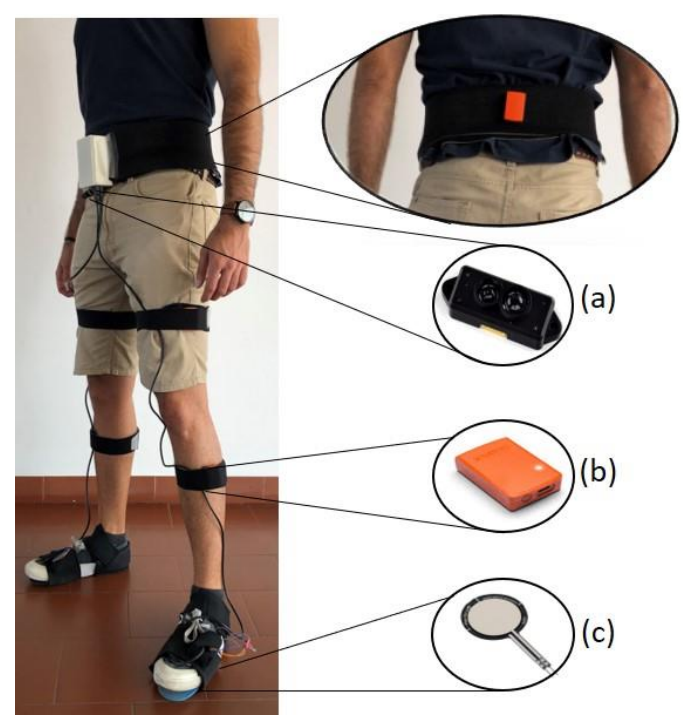

Figure 2. Wearable Motion LAB sensor placement. (a) Infrared laser sensor. (b) Xsens IMU sensor. (c) FSR ground reaction force sensor

The proposed work approached two main research questions, as follows: (i) what are the best gait measures for LMs and LM transition classification? and (ii) Would the environment-aware measure help to increase LM and LM transition prediction performance? These questions are explored in Section III.

\section{METHODS}

The LM and LM transition recognition and prediction tool proposed in this dissertation is represented in Fig.1. The proposed methodology is divided into five main modules, namely the wearable motion LAB (WML), biomechanical model, musculoskeletal model, feature computing and the machine learning framework.

\section{A. Wearable Motion LAB}

The WML includes ergonomic, stand-alone, wearable sensor systems placed on the subject's body, namely the infrared laser sensor, the inertial sensor system and the force sensing resistor (FSR) system. The first sensor refers to the

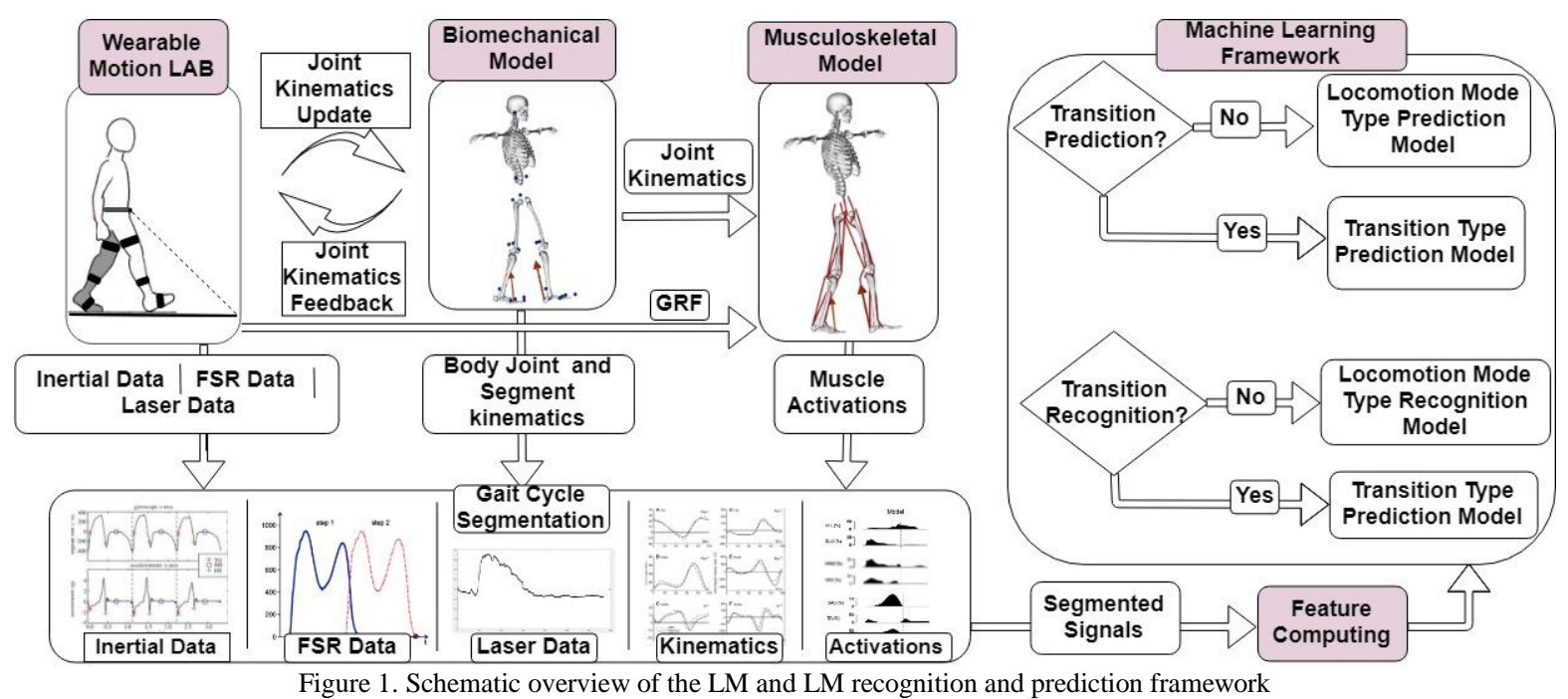

Figure 1. Schematic overview of the LM and LM recognition and prediction framework 
infrared laser sensor (TF Mini LiDAR), placed on the user waist pointing at the ground with and angle of proximally $45^{\circ}$ to the user's abdomen vertical plane (Fig. 1 (a)). This laser sensor provides a signal that indicates the distance between the subject and the ground or possible obstacles and terrain changes. The second system is composed by 7 wireless IMU (Xsens Technologies BV, Enschede, Netherlands) placed on the outer side of both thighs and shanks, on top of the feet, and one IMU was placed on the lower back (Fig. 2(b)). The IMUs provide acceleration, angular velocity and magnetic field regarding each lower limb segment. The third system consists of 4 FSRs (406 FSR, Interlink Electronics), two per foot, one placed in the and one in the heel. The FSRs provide an electrical signal that is directly related to the pressure applied, measuring the ground reaction force (GRF). All systems were synchronized by wires and the data was collected at $100 \mathrm{~Hz}$.

\section{B. Biomechanical Model}

The biomechanical model is accountable for the 3D joint angle estimations using IMUs' measures as input. The Xsens well-established motion tracking method, was used to guarantee accurate joint angle estimations, as the lower limb joint angles represent a significative feature of the human gait, and consequently, they are relevant for LM and LMT classification [15]. The Xsens biomechanical model is in constant communication with the wireless IMUs, by sending them the real body segment positions and simultaneously receiving new acquisitions to predict the subsequent motions.

\section{Musculoskeletal Model}

The third module of the tool depicted in Fig.1 includes a virtual musculoskeletal model able to compute the motion forces and muscle activations involved in locomotion from the given kinematic data (lower limb spatial displacement) and the force of interaction with the external world (from the FSRs). Since during locomotion, the subject only contacts the ground, the GRF (from the FSRs) is the only considered external force applied.

The virtual model consists of a modified version of the gait10dof18musc OpenSim model, which includes the trunk, pelvis, and leg segments with 10 degrees of freedom. The musculoskeletal system incorporates 18 Hill-type muscles (9 in each leg), where 5 muscles are monoarticular (Soleus, Tibialis Anterior, Gluteus Maximus, Vastus Intermedius, Rectus Femoris) and 3 muscles are biarticular (Gastrocnemius, Biceps Femoris, Lateral Hamstring, Iliopsoas). Literature suggests that the used musculoskeletal system underlies the sufficient characteristics to impose a natural human gait, capable of tolerating small terrain disturbances, being computationally efficient [18].

The motion forces (e.g., net forces and torques) at each joint are computed through inverse dynamics. The algorithm solves the dynamic equation of motion presented in (1), where $q, \dot{q}$ and $\ddot{q}$ refer to the position, velocity, and acceleration of the generalized coordinates; $M, C, G$ are the mass, centrifugal and Coriolis, and gravitational forces matrices, respectively; and $T$ is the vector of unknown motion forces. This algorithm derives the minimal forces responsible for generating the desired motion.

$$
M(q) \ddot{q}+C(q, \dot{q})+G(q)=T
$$

With the purpose of computing the muscle activations, a static optimization algorithm was applied to compute muscle activations by minimizing its sum of squared.

\section{Feature Computing}

The feature computing module converts the input sensor and neuro-biomechanical data, to an output feature vector containing meaningful information segmented per gait cycle. The time window's size is adaptive according to the gait speed. The period of each time-window corresponds to the duration of each gait cycle. The gait cycle segmentation was determined by automatically detect gait events using an algorithm based on the angular velocity of each foot [19].

The developed framework performs both LM and LM transition recognition and prediction. For recognition purposes, gait cycles were segmented between consecutive heel-strike events while for prediction purposes, gait cycles were segmented between consecutive toe-off events.

For neuro-biomechanical data, several features were computed such as the mean, standard deviation, range, maximum, minimum and the first and last value. Regarding the laser sensor data, some additional features were computed with the purpose of taking maximum advantage of the signal characteristics, namely, the signal slope, slope module, previous slope, and previous slope module. Table I lists the computed features per gait measure.

TABLE I. FEATURE COMPUTING REPRESENTATION CONSIDERING SENSOR AND NEURO-BIOMECHANICAL DATA

\begin{tabular}{|c|c|c|c|}
\hline Sensor/Tool & Measure & Body Zone & Features \\
\hline IMU & $\begin{array}{l}\text { Angular } \\
\text { Velocity }\end{array}$ & $\begin{array}{l}\text { Back, right/left Thigh, } \\
\text { right/left Shank and } \\
\text { right left foot }\end{array}$ & \multirow{4}{*}{$\begin{array}{l}\text { Mean, Standard } \\
\text { Deviation, Range } \\
\text { of Motion, } \\
\text { Maximum, } \\
\text { Minimum and the } \\
\text { First and Last } \\
\text { Value }\end{array}$} \\
\hline $\begin{array}{c}\text { Xsens } \\
\text { Biomechanical } \\
\text { Model }\end{array}$ & Joint Angles & $\begin{array}{l}\text { Right/Left Hip, } \\
\text { Right/Left Knee, } \\
\text { Right/Left Ankle }\end{array}$ & \\
\hline $\begin{array}{l}\text { Opensim } \\
\text { Muscluskeletal } \\
\text { Model }\end{array}$ & $\begin{array}{c}\text { Muscle } \\
\text { Activations }\end{array}$ & $\begin{array}{l}\text { Soleus, Tibialis Ant., } \\
\text { Gluteus Max., Vastus } \\
\text { Int., Rectus Fem, } \\
\text { Gastrocnemius, Biceps } \\
\text { Fem., Lateral Ham., } \\
\text { Iliopsoas }\end{array}$ & \\
\hline FSRs & $\begin{array}{l}\text { Ground } \\
\text { Reaction } \\
\text { Force }\end{array}$ & Right/left Heel and Toe & \\
\hline Laser & $\begin{array}{l}\text { Distance } \\
\text { between } \\
\text { subject and } \\
\text { ground }\end{array}$ & Waist & $\begin{array}{c}\text { Mean, Standard } \\
\text { Deviation, Range } \\
\text { of Motion, } \\
\text { Maximum, } \\
\text { Minimum and the } \\
\text { First and Last } \\
\text { Value. } \\
\text { Slope, Slope } \\
\text { Module, Previous } \\
\text { Slope and } \\
\text { Previous Slope } \\
\text { Module }\end{array}$ \\
\hline
\end{tabular}

\section{E. Machine Learning Framework}

The machine learning framework aims at recognizing and predicting LM and LM transitions to infer about the subject's locomotion intentions in diverse terrain types encountered in daily life. This machine learning framework unfolds in four sub-sections, namely, Data Pre-processing, Data Labelling, Model Building, and Classification Evaluation. 


\section{1) Data Pre-processing}

Pre-processing techniques are applied to the unprocessed feature vector in order to maximize model performance and reduce its training time. The applied pre-processing techniques include data normalization and feature selection. During data normalization, features are processed using the min-max scaling method. This process aims to convert all features to a common range such that features with larger value range do not reduce the influence of features with smaller ranges. The min-max method provides zero-mean and unit-variance characteristics to each feature and limits range between -1 and 1 .

Additionally, a feature selection method was used to select the minimum number of features. For this purpose, the mRMR plus forward selection method was used. This method selects the most relevant features from those that have minimally redundancy, i.e. selects features that are maximally dissimilar to each other. This method creates a ranking immune to redundancy, in which a feature is kept if it increases the tool's performance, otherwise it is removed [20].

\section{2) Data Labelling}

For recognition and prediction purposes, labelling was done on features computed considering time-windows segmented between heel strike events and toe off events, respectively. Moreover, prediction was achieved by classifying events one gait cycle before it's occurrence (i.e., on the previous time window as demonstrates by blue timezones in Fig. 3). An example of the time displacement of recognition (red) and prediction time-windows (blue) used for labelling and feature computing, are represented in Fig. 3.

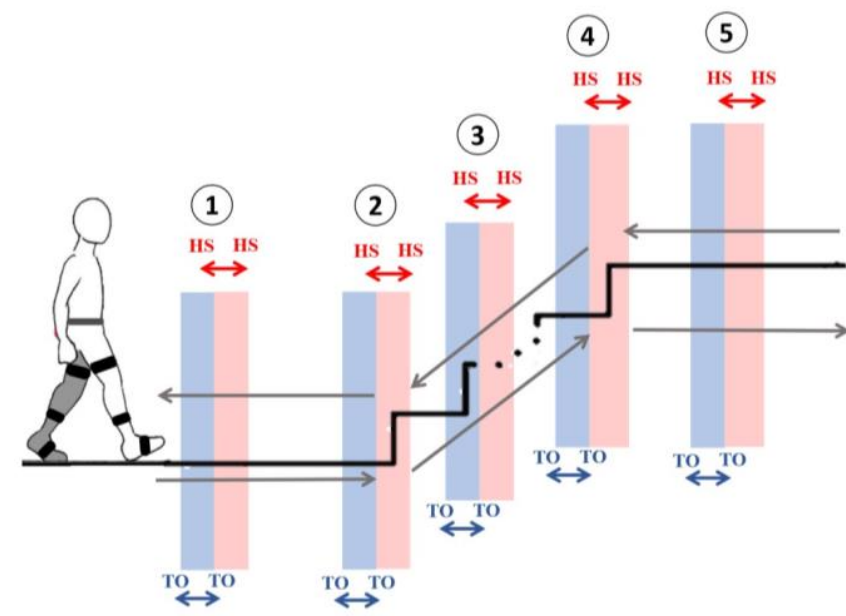

Figure 3. Considered time-windows for LM (1, 3 and 5) and LM transition ( 2 and 4$)$ recognition (red) and prediction (blue). Prediction timewindows are limited by toe-off events (TO) and recognition time windows are limited by heel strike events

\section{3) Model Building}

The followed approach assumes that the decision making is empowered by four classification models for recognition and another four for prediction running simultaneously. Fig. 1 depicts a simplified scheme of this classification workflow. The classification procedure was constructed as a chain sequence of classification models as demonstrated in Fig. 4.

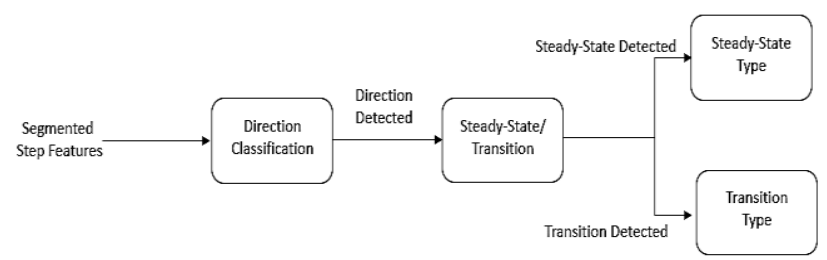

Figure 4. Classification chain sequence

The workflow starts with the walking direction classification using the Direction Classification Model. This classification model aims at classifying forward, anticlockwise and clockwise directions. The remaining classification sequence only proceeds if forward direction is detected. The following classification model is the binary Steady-State/Transition model and it aims at distinguishing LM steady-states (i.e. during a certain LM) from LM transition states (during an LM change), regardless of what LM or LM transition type it represents. In case this classification model detects a steady-state, it proceeds to the Steady-State Type model to determine its type. The SteadyState Type model classifies five LM, namely, level-walking, stair ascend/descend, and ramp ascends/descend. On the other hand, if the Steady-State Transition detects an LM transition, it proceeds to the Transition Type model to determine the transition type. The Transition Type model classifies eight transitions from level ground to stair/ramp ascend/descend and from to stair/ramp ascend/descend to level ground. To note that this chain sequence of classification is used for both recognition and prediction purposes.

This scheme allows the easy incorporation of additional LM and/or LM transition in the classification, adding versatility to the proposed framework to act as a benchmark tool. Additionally, it seems to be advantageous regarding the classification strategy proposed by Young et al. [21] since it demands the implementation of fewer models, decreasing the computational load and the susceptibility to sequential errors, and allows easier integration of further LMs and/or LM transition.

The SVM classifier with a Gaussian kernel was used for all classification models. A hyperparameter optimization was performed to tune the kernel box constraint (C) and scale parameter $(\sigma)$. The hyperparameter optimization involved a grid search to determine the combination the yielded the highest performance. This process was conducted by building a model for each combination, performing its evaluation iteratively. The values for each hyperparameter varied according to a range that goes from from $2^{-10}$ to $2^{10}$. To note that all classification models are built considering features retrieved from all users which conveys a user-independent characteristic.

\section{4) Classification Evaluation}

The classification evaluation was conducted using crossvalidation. The evaluation procedure is used for comparing models using different input parameters such as varying hyperparameters or feature combinations. The evaluation is particularly important to infer the classification performance of unseen data. Each model's performance is evaluated using repeated 10-fold cross-validation, repeated 10 times. To evaluate the classification results, the Matthew's correlation 
coefficient (MCC) was used due to its good representative properties of unbalanced classes [22], as for the case of this work. The accuracy (ACC) was also computed for comparing the results with the literature's findings [23].

\section{F. Experimental Conditions}

The experimental trials were performed with 10 healthy subjects ( 7 males and 3 females with a mean age of $23.9 \pm$ 1.64 years, mean height of $174.9 \pm 7.07 \mathrm{~cm}$ and mean weight of $69.7 \pm 7.14 \mathrm{~kg}$ ). The participants were asked to ascend and descend a ramp and ascend and descend a staircase 5 times per each terrain elevation (total of 20 terrain elevations per subject). In these trials, subjects started on level-terrain then transited to the ramp or staircase, walk the full ramp/staircase length and back to level-terrain walking. When in levelterrain, the users walked 3-4 m. The staircase had 8 steps with $17 \mathrm{~cm}$ of height, $31 \mathrm{~cm}$ of depth and $110 \mathrm{~cm}$ width. The ramp was $10.3 \mathrm{~m}$ with a 10 degrees inclination. Fig. 5 depicts the terrain's conditions for level, ramp and stair walking. The subjects could freely perform the LM transitions with any leading leg to enable transition seamlessly and intuitively between LMs. To note that the participants were always instrumented with all sensors that constitute the wearable motion LAB.

As ground truth, an assessor marked the LM transition instants during the experimental trials using a button synchronized with the system.

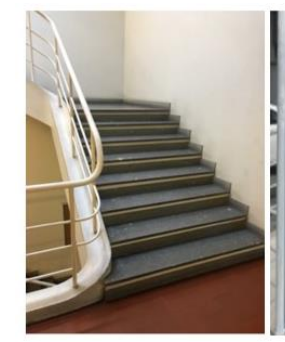

(A)

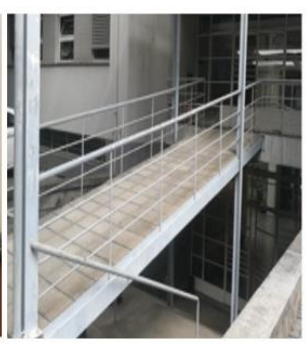

(B)

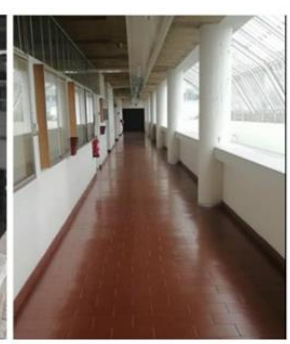

(C)
Figure 5. Pictures of the terrain conditions used in the trials (a) staircase, (b) slope terrain, (c) level ground terrain

\section{RESUltS AND DisCUSSION}

In order to tackle these dissertation goals, three studies were performed combining a specific group of gait measures, namely, (i) biomechanical measures, (ii) neuro-biomechanical measures, and (iii) an environment-aware/biomechanical based measures. All the mentioned studies approached the LM and LM transition recognition and prediction.

The first study aimed to determine if features computed from gait measures (joint angles and angular velocity at sagittal plane) of the Xsens system alone provide good performance. The results obtained from this study are demonstrated in Table II for recognition and prediction. For this study, a total of 66 features were computed and subsequently pre-processed.
TABLE II. BIOMECHANICAL BASED FEATURE RECOGNITION AND PREDICTION

\begin{tabular}{|c|c|c|c|c|c|}
\hline & $\begin{array}{c}\text { Classification } \\
\text { Model }\end{array}$ & $\begin{array}{l}\text { Hyperpa } \\
\text { rameters }\end{array}$ & $\begin{array}{c}N^{o} \\
\text { Selected } \\
\text { features }\end{array}$ & $M C C$ & $A C C$ \\
\hline \multirow{3}{*}{ Recognition } & $\begin{array}{c}\text { Steady- } \\
\text { State/Transition }\end{array}$ & \multirow{6}{*}{$\begin{aligned} C & =64 \\
\sigma & =4\end{aligned}$} & 34 & $\begin{array}{c}0.873 \pm \\
0.008\end{array}$ & $\begin{array}{c}0.957 \pm \\
0.003\end{array}$ \\
\hline & Transition Type & & 23 & $\begin{array}{c}0.922 \pm \\
0.041\end{array}$ & $\begin{array}{c}0.982 \pm \\
0.01\end{array}$ \\
\hline & $\begin{array}{c}\text { Steady-State } \\
\text { Type }\end{array}$ & & 24 & $\begin{array}{c}0.989 \pm \\
0.004 \\
\end{array}$ & $\begin{array}{c}0.997 \pm \\
0.001 \\
\end{array}$ \\
\hline \multirow{3}{*}{ Prediction } & $\begin{array}{c}\text { Steady- } \\
\text { State/Transition }\end{array}$ & & 27 & $\begin{array}{l}0.84 \pm \\
0.006\end{array}$ & $\begin{array}{c}0.948 \pm \\
0.002 \\
\end{array}$ \\
\hline & Transition Type & & 22 & $\begin{array}{c}0.83 \pm \\
0.04\end{array}$ & $\begin{array}{c}0.958 \pm \\
0.01\end{array}$ \\
\hline & $\begin{array}{l}\text { Steady-State } \\
\text { Type }\end{array}$ & & 23 & $\begin{array}{c}0.948 \pm \\
0.05 \\
\end{array}$ & $\begin{array}{c}0.984 \pm \\
0.02 \\
\end{array}$ \\
\hline
\end{tabular}

From the observation of Table II, it is possible to acknowledge that all classification models show high recognition and prediction performance (ACC>0.948). However, the MCC values for recognition and prediction of the steady-state/transition classification model are a little bellow when comparing with the remaining $(\mathrm{MCC}=0.8729$ and $\mathrm{MCC}=0.8482$ respectively). Furthermore, the recognition performance was higher than the prediction performance for the considered classification models. These results point out that the use of biomechanical features may be insufficient to recognize and predict LM transition and are most suited for recognition purposes. The findings are similar with those reported in the literature, that only used IMU-based features, as seen in the work proposed by Jang et. al [5],that reported an ACC of 0.98, 0.95 and 0.99 for SA, SD, and LG, respectively. Nonetheless, the proposed work addressed prediction problematic, in opposition to [5]

The second study aimed to determine the influence of adding neuromuscular features (computed from muscle activation patterns of 18 muscles of Opensim model) to the biomechanical features used in the first study. The results obtained from this study are demonstrated in Table III for recognition and prediction This study used a total of 156 features combining he 66 biomechanical features to the 90 neuromuscular features.

TABLE III. NEIRO-BIOMECHANICAL (NEUROMUSCULAR PLUS BIOMECHANICAL) FEATURE RECOGNITION AND PREDICTION

\begin{tabular}{|c|c|c|c|c|c|}
\hline & $\begin{array}{c}\text { Classification } \\
\text { Model }\end{array}$ & $\begin{array}{l}\text { Hyperpa } \\
\text { rameters }\end{array}$ & $\begin{array}{c}N^{o} \\
\text { Selected } \\
\text { features }\end{array}$ & $M C C$ & $A C C$ \\
\hline \multirow{3}{*}{ Recognition } & $\begin{array}{c}\text { Steady- } \\
\text { State/Transition }\end{array}$ & \multirow{6}{*}{$\begin{aligned} \mathrm{C} & =64 \\
\sigma & =4\end{aligned}$} & 29 & $\begin{array}{c}0.867 \pm \\
0.01\end{array}$ & $\begin{array}{c}0.955 \pm \\
0.005\end{array}$ \\
\hline & Transition Type & & 21 & $\begin{array}{c}0.961 \pm \\
0.02\end{array}$ & $\begin{array}{c}0.996 \pm \\
0.003\end{array}$ \\
\hline & $\begin{array}{c}\text { Steady-State } \\
\text { Type }\end{array}$ & & 22 & $\begin{array}{c}0.949 \pm \\
0.02 \\
\end{array}$ & $\begin{array}{c}0.985 \pm \\
0.006 \\
\end{array}$ \\
\hline \multirow{3}{*}{ Prediction } & $\begin{array}{c}\text { Steady- } \\
\text { State/Transition }\end{array}$ & & 16 & $\begin{array}{c}0.847 \pm \\
0.01\end{array}$ & $\begin{array}{c}0.947 \pm \\
0.004\end{array}$ \\
\hline & Transition Type & & 24 & $\begin{array}{c}0.871 \pm \\
0.03\end{array}$ & $\begin{array}{c}0.985 \pm \\
0.005\end{array}$ \\
\hline & $\begin{array}{l}\text { Steady-State } \\
\text { Type }\end{array}$ & & 24 & $\begin{array}{c}0.921 \pm \\
0.01\end{array}$ & $\begin{array}{c}0.975 \pm \\
0.004\end{array}$ \\
\hline
\end{tabular}

For this study, it was hypothesized that the muscle activation patterns would provide a significant contribution for predicting LM transitions since these signals have the purpose of controlling muscle activation that generates the movement of the subject [24]. The general appreciation of the obtained results is that the additional neuromuscular features provided a similar performance when compared to the only 
use of biomechanical features (first study). Although the prediction performance for the Steady-State/Transition model stayed in line with the first study performance, it is noticeable that in this second study, 11 fewer features were used. This finding indicates that it needed much less information to achieve a similar result. Therefore, despite not improving performance considerably, neuromuscular features present meaningful information regarding the LM and LM transition classification.

The presented results stand in line with the literature. Huang et. al.[8] fused biomechanical sensors with EMG features features and reported an ACC value of proximally 0.978 in LM recognition whereas the herein developed tool rounded an ACC value of 0.995. Moreover, Huang's work predicted LM transitions with an ACC of 1 for similar terrains, which is higher than the 0.947 ACC value obtained. However, is important to mention that in Huang's work the transition steps were not performed with the leading leg. Furthermore, the work developed by Tkarch et. al. [9] reported an higher ACC (0.977) relatively to the proposed work (0.947) when using EMG data fused with biomechanical data for transition prediction. Nonetheless, both works used biomechanical prosthesis signals which are characterized for being more "robotic" when comparing to walking patterns of different subjects.

The third study tested the LM and LM transition recognition and prediction performance when using biomechanical (GRF data, IMU sagittal angular velocity, lower limb sagittal joint angles) and environment-aware (infrared laser) features. The results obtained from this study are presented in Table IV for recognition and prediction. This study used a total of 100 features.

TABLE IV. ENVIRONMENT-AWARE/BIOMECHANICAL BASED FEATURE RECOGNITION AND PREDICTION

\begin{tabular}{|c|c|c|c|c|c|}
\hline & $\begin{array}{c}\text { Classification } \\
\text { Model }\end{array}$ & $\begin{array}{l}\text { Hyperpa } \\
\text { rameters }\end{array}$ & $\begin{array}{c}N^{o} \\
\text { Selected } \\
\text { features }\end{array}$ & $M C C$ & $A C C$ \\
\hline \multirow{4}{*}{ Recognition } & Direction & \multirow{8}{*}{$\begin{array}{c}\mathrm{C}=64 \\
\sigma=4\end{array}$} & 33 & $\begin{array}{c}0.995 \pm \\
0.01\end{array}$ & $\begin{array}{c}0.998 \pm \\
0.002\end{array}$ \\
\hline & $\begin{array}{c}\text { Steady- } \\
\text { State/Transition }\end{array}$ & & 57 & $\begin{array}{c}0.887 \pm \\
0.02 \\
\end{array}$ & $\begin{array}{c}0.947 \pm \\
0.01 \\
\end{array}$ \\
\hline & Transition Type & & 19 & $\begin{array}{c}0.959 \pm \\
0.005\end{array}$ & $\begin{array}{c}0.990 \pm \\
0.004\end{array}$ \\
\hline & $\begin{array}{l}\text { Steady-State } \\
\text { Type } \\
\end{array}$ & & 20 & $\begin{array}{c}0.989 \pm \\
0.01 \\
\end{array}$ & $\begin{array}{c}0.983 \pm \\
0.002 \\
\end{array}$ \\
\hline \multirow{4}{*}{ Prediction } & Direction & & 52 & $\begin{array}{c}0.997 \pm \\
0.02\end{array}$ & $\begin{array}{c}0.999 \pm \\
0.001\end{array}$ \\
\hline & $\begin{array}{c}\text { Steady- } \\
\text { State/Transition }\end{array}$ & & 51 & $\begin{array}{c}0.865 \pm \\
0.03\end{array}$ & $\begin{array}{c}0.945 \pm \\
0.004\end{array}$ \\
\hline & Transition Type & & 36 & $\begin{array}{c}0.978 \pm \\
0.02\end{array}$ & $\begin{array}{c}0.995 \pm \\
0.005\end{array}$ \\
\hline & $\begin{array}{l}\text { Steady-State } \\
\text { Type }\end{array}$ & & 37 & $\begin{array}{c}0.949 \pm \\
0.005 \\
\end{array}$ & $\begin{array}{c}0.983 \pm \\
0.002\end{array}$ \\
\hline
\end{tabular}

Regarding third study, a direction classification model was included, and it demonstrated excellent performance both for recognition and prediction purposes (ACC $=0.998$ and $\mathrm{ACC}=0.999$, respectively). This finding indicates that the information given to the model was adequate to characterize forward, anticlockwise, and clockwise direction of a walking subject. Novak et. al [25] proposed a turn detection system using IMUs and reported results similar to the ones achieved in this work for direction prediction $(\mathrm{ACC}>0.97 \mathrm{vs} \mathrm{ACC}=$ 0.95 , respectively).
Furthermore, the combination of GRF and laser-based features to the biomechanical features increased the performance for all classification models in recognition and prediction. Despite performance improvement using the mentioned additional features, the GRF number of features selected is very low. In this sense, the added laser data features represent higher importance in classification as they were the major reason for the classification performance improvement regarding recognition and prediction. The highest increase was related to the recognition and prediction of the type of transition (MCC $=0.959$ and $\mathrm{MCC}=0.978$, respectively). These results might be related to the abrupt changes in the laser signals characteristics when a terrain change occurs. Features computed considering these laser characteristics may provide meaningful information to classify transition types, as observed in a team study [26]. The fusion between biomechanical sensors with laser data was already explored in literature by Liu et. al.[17], reported an ACC of 99\% in LM recognition, equal to the achieved $99 \%$ in ACC. Nonetheless, LM transitions were not considered in [17], as innovatively explored in this work.

In summary, the Direction classification model presented in Environment-Aware/Biomechanical based feature study revealed excellent results $(\mathrm{ACC}=0.998$ and $\mathrm{ACC}=0.999$ ) regarding recognition and prediction respectively. Similar finding can be found in [25] with a success rate of 0.97 to 0 . 99 in ACC values. The Steady-State/Transition classification models revealed the lowest general performance which might be related to the characteristic of having to distinguish between only two classes (binary classification), and because it was trained in an unbalanced way (fewer LM transition features). The Transition Type and Steady-State Type Classification models have shown good classification performance in a general manner.

\section{CONCLUSION}

This dissertation proposes an automatic and userindependent machine learning-based tool to recognize and predict daily performed LMs and LM transitions. The work aimed to determine the combination of gait measures collected by wearable sensors that yielded the best classification performance.

The findings showed that the automatic recognition and prediction tool demonstrated the highest performance for environment-aware/biomechanical based feature combination. Moreover, since GRF-based features provided poor significance, it is possible to conclude that the best feature combination for LM and LM transition classification includes biomechanical features from Xsens together with environment-aware based features from the laser sensor.

Furthermore, the environment-aware features demonstrated to be relevant to classify LM transitions, namely they increased the LM transition prediction performance.

These findings contribute to identifying the meaningful gait measures for building an effective user's motion intent recognition. This will allow a natural and smooth response of lower limb active assistive devices according to the user's intentions and needs. 


\section{REFERENCES}

[1]

"Spinal cord injury," 2013. [Online]. Available: https://www.who.int/news-room/fact-sheets/detail/spinal-cordinjury. [Accessed: 07-Mar-2019].

[2] World Healthy Organization (WHO), "Global burden of stroke," atlas Hear. Dis. stroke, vol. 15, pp. 50-51, 2004.

[3] M. R. Tucker et al., "Control strategies for active lower extremity prosthetics and orthotics: a review," J. Neuroeng. Rehabil., vol. 12, no. 1, p. 1, Jan. 2015.

[4] Y. D. Li and E. T. Hsiao-Wecksler, "Gait Mode Recognition and Control for a Portable - Powered Ankle - Foot Orthosis," 2013.

[5] J. Jang, K. Kim, J. Lee, B. Lim, and Y. Shim, "Online gait task recognition algorithm for hip exoskeleton," IEEE Int. Conf. Intell. Robot. Syst., vol. 2015-Decem, pp. 5327-5332, 2015.

[6] Y. He, D. Eguren, T. P. Luu, and J. L. Contreras-Vidal, "Risk management and regulations for lower limb medical exoskeletons: A review," Med. Devices Evid. Res., vol. 10, pp. 89-107, 2017.

[7] B. Chen, E. Zheng, and Q. Wang, "A locomotion intent prediction system based on multi-sensor fusion," Sensors (Switzerland), vol. 14, no. 7, pp. 12349-12369, 2014.

[8] H. Huang, F. Zhang, L. J. Hargrove, Z. Dou, D. R. Rogers, and K. B. Englehart, "Continuous locomotion-mode identification for prosthetic legs based on neuromuscular - Mechanical fusion," IEEE Trans. Biomed. Eng., vol. 58, no. 10, pp. 2867-2875, 2011.

[9] D. C. Tkach and L. J. Hargrove, "Neuromechanical sensor fusion yields highest accuracies in predicting ambulation mode transitions for trans-tibial amputees," Proc. Annu. Int. Conf. IEEE Eng. Med. Biol. Soc. EMBS, pp. 3074-3077, 2013.

[10] E. M. Diaz, F. De Ponte Muller, A. R. Jimenez, and F. Zampella, "Evaluation of AHRS algorithms for inertial personal localization in industrial environments," Proc. IEEE Int. Conf. Ind. Technol., vol. 2015-June, no. June, pp. 3412-3417, 2015.

[11] M. D. Djurić-Jovičić, N. S. Jovičić, and D. B. Popović, "Kinematics of gait: New method for angle estimation based on accelerometers," Sensors, vol. 11, no. 11, pp. 10571-10585, 2011.

[12] K. Lebel, P. Boissy, M. Hamel, and C. Duval, "Inertial measures of motion for clinical biomechanics: Comparative assessment of accuracy under controlled conditions - Changes in accuracy over time," PLoS One, vol. 10, no. 3, pp. 1-12, 2015.

[13] Y. David Li and E. T. Hsiao-Wecksler, "Gait mode recognition and control for a portable-powered ankle-foot orthosis," IEEE Int. Conf. Rehabil. Robot., 2013.

[14] A. J. Young, T. A. Kuiken, and L. J. Hargrove, "Analysis of using EMG and mechanical sensors to enhance intent recognition in powered lower limb prostheses," J. Neural Eng., vol. 11, pp. 1-12, 2014.

[15] D. Roetenberg, H. Luinge, and P. Slycke, "Xsens MVN : Full 6DOF Human Motion Tracking Using Miniature Inertial Sensors," Hand, The, no. January 2009, pp. 1-7, 2009.

[16] C. Pizzolato et al., "CEINMS: A toolbox to investigate the influence of different neural control solutions on the prediction of muscle excitation and joint moments during dynamic motor tasks," J. Biomech., vol. 48, no. 14, pp. 3929-3936, 2015.

[17] M. Liu, D. Wang, and H. Helen Huang, "Development of an Environment-Aware Locomotion Mode Recognition System for Powered Lower Limb Prostheses," IEEE Trans. Neural Syst. Rehabil. Eng., vol. 24, no. 4, pp. 434-443, 2016.

[18] H. Geyer and H. Herr, "A Muscle-Reflex Model that Encodes Principles of Legged Mechanics Produces Human Walking Dynamics and Muscle Activities," 2010.

[19] P. Félix, J. Figueiredo, C. P. Santos, and J. C. Moreno, "Adaptive real-time tool for human gait event detection using a wearable gyroscope," 20th Int. Conf. Climbing Walk. Robot. Support Technol. Mob. Mach. (CLAWAR 2017), pp. 1-9, 2017.

[20] Y. Zhang, C. Ding, and T. Li, "Gene selection algorithm by combining reliefF and mRMR.," BMC Genomics, vol. 9 Suppl 2, p. S27, 2008.

[21] A. J. Young and L. J. Hargrove, “A Classification Method for UserIndependent Intent Recognition for Transfemoral Amputees Using Powered Lower Limb Prostheses," IEEE Trans. Neural Syst. Rehabil. Eng., vol. 24, no. 2, pp. 217-225, 2016.

[22] G. Jurman, S. Riccadonna, and C. Furlanello, "A comparison of MCC and CEN error measures in multi-class prediction," PLoS
One, vol. 7, no. 8, pp. 1-8, 2012.

[23] J. Figueiredo, C. P. Santos, and J. C. Moreno, "Automatic recognition of gait patterns in human motor disorders using machine learning: A review," Med. Eng. Phys., vol. 53, pp. 1-12, 2018.

[24] T. Afzal, K. Iqbal, G. White, and A. B. Wright, "A Method for Locomotion Mode Identification Using Muscle Synergies," IEEE Trans. Neural Syst. Rehabil. Eng., vol. 25, no. 6, pp. 608-617, 2017.

[25] D. Novak, M. Goršič, J. Podobnik, and M. Munih, "Toward RealTime Automated Detection of Turns during Gait Using Wearable Inertial Measurement Units," Sensors, vol. 14, no. 10, pp. 18800$18822,2014$.

[26] S. Carvalho, J. Figueiredo, and C. P. Santos, "Environment-Aware Locomotion Mode Transition Prediction System," in 2019 IEEE International Conference on Autonomous Robot Systems and Competitions (ICARSC), 2019, pp. 1-6. 